service in many ways. The volumes altogether are very readable, and throughout the statements are usually reliable. Should a second edition be required it might possibly be improved by the addition of a "table of contents."

W. H. W.

\section{THE GERM THEORY}

The Germ Theory Applied to the Explanation of the Phenomena of Disease. "The Specific Fevers." By T. Maclagan, M.D. (London: Macmillan and Co., 1876.)

T $N$ his preface the author states that "one object which 1 he has in view is to rescue the germ theory of disease from a false and misleading position, and to give to it its true and legitimate standing as a pathological question." The subject discussed is whether the propagation of germs in the system can produce specific fevers. He believes it can, and assumes that all contagia are living organisms, probably albuminous, reproducing their kind, living for a considerable period, speedily perishing when freely exposed to the atmosphere, and so minute as to elude the highest powers of the microscope.

If, however, the particles in sheep-pox, small-pox, and vaccine, be the infecting matter, they are easily seen by the microscope, and ought therefore to be found in the blood, but such is not the case.

Dr. Maclagan holds that "all microzymes are not contagia, but all contagia may be microzymes." The fact that the contagia fluids are most potent when fresh, and that their virulence diminishes as bacteria increase in them is explained by saying that disease-germs are more minute organisms than bacteria, and are the food on which bacteria live. According to this view, bacteria not only do not constitute infection, but destroy it.

Dr. Maclagan says "that the chief action of an organism on its environment is the consumption of nitrogen and water. A disease-germ is a parasite, and requires a special nidus as well as nitrogen and water; the parasite finds a something in its nidus-the second factorthe parasite being the first. Without this second factor no bad result follows the reception of the contrgium." Different periods of incubation are accounted for by the varying amount of the second factor and the number of germs imbibed; incubation itself by germ-growth and reproduction; and the onset of the symptoms by the germs becoming mature.

The consumption of nitrogen by the contagium particles causes wasting of the tissues, i.e., the organisms eat the albumen intended to nourish the body. They also drink largely of water from the liquor sanguinis, which, being rich in soda, explains why soda-salts are often absent from the excretions during fever. The same retention of soda-salts, however, often happens in acute pneumonia, which has no relation to infectious disease.

Increased elimination of urea is explained thus:"The increased consumption of liquor sanguinis by the contagium particles leads to increased formation of retrogressive albumen and of urea." It seems by this that contagium particles have livers and kidneys, and excrete urea. Diminished excretion of urea is held as "due to consumption by the contagium particles of the water requisite to enable the kidneys to perform their excretory function." What then, we ask, becomes of the water consumed by the contagium particles? Do their kidneys excrete urea in excess with limited water, while those of the patient are unable to do so? or do the contagium particles not consume water when the urea is increased as well as when it is diminished? In fever the quantity of water drank by the patients is very great, but that, according to Dr. Maclagan, is because the quantity of contagium particles is also very great. We find, however, the same symptoms in symptomatic fevers, with no contagium. particles present, and we have great difficulty in believing that ultra-microscopic organisms in a person's blood could consume several tumblerfuls of water in twenty-four hours.

The heat of specific fevers is partly ascribed to the propagation of the contagium causing increased consumption of tissue. But increase of living matter causes the disappearance of heat, not its production. Again, the author states that the fecundation of the organisms may be accompanied by an elevation of temperature analogous to that which occurs under similar circumstances in other organisms.

As regards treatment, at page 163 the following occurs : "If we were to bleed, to purge, to give antimony to, or even simply to withhold food and water from, all the cases of typhus and enteric fever which occur, there can be no doubt that we should find the mortality from those diseases greatly increased." Dr. Maclagan is right here, for by simply withholding food and water, there can be no doubt that he would greatly increase the mortality by starving his patients to death. He, however, believes that fever patients should be supplied with nitrogen and water to compensate for what the organisms consume.

The cessation of the fever and its specificity are attributed-the first, to the organisms, as parasites, requiring a special nidus which contains a suitable pabulum, and when the latter is exhausted the fever ceases; the second, to a local lesion in the nidus, which is the part where fecundation of the organism takes place. In small pox this nidus and lesion is in the skin, in typhoid in the bowels, and so on. It is well-known, however, that many medicines act on special parts of the body, and yet we do not think of calling them parasites which require a special nidus. The author gives many other plausible, and some very unusual explanations of febrile phenomena by means of the germ theory, all of which, we believe, are far more clearly and rationally explicable on the physicochemical theory. The writing of this book must have cost Dr. Maclagan much trouble. We have read it very carefully, and commend the author's honesty in stating his views, but question if the work will go far in realising the object for which it was ostensibly written.

\section{OUR BOOK SHELF}

A Manual of Cinchona Cultivation in India. By George King, M.B., F.L.S., Superintendent of the Royal Botanical Garden, Calcutta, and of Cinchona Cultivation in Bengal. (Calcutta, 1876.)

THIs manual is another contribution to the numerous books, papers, and articles that have appeared of late years on the subject of cinchona. Varied as these contributions have been, and valuable each one in itself, this manual brings together much that is useful, not only on 